\title{
Energy, entropy and mass scaling relations for elliptical galaxies. Towards a physical understanding of their photometric properties
}

\author{
I. Márquez ${ }^{1}$, G. B. Lima Neto $^{2}$, H. Capelato ${ }^{3}$, F. Durret ${ }^{4,5}$, B. Lanzoni ${ }^{4,6}$, and D. Gerbal ${ }^{4,5}$ \\ 1 Instituto de Astrofísica de Andalucía (C.S.I.C.), Apartado 3004, 18080 Granada, Spain \\ 2 Instituto Astronômico e Geofísico/USP, Av. Miguel Stefano 4200, São Paulo/SP, Brazil \\ 3 Instituto Nacional de Pesquisas Espaciais, São José dos Campos/SP, Brazil \\ 4 Institut d'Astrophysique de Paris, CNRS, 98bis Bld Arago, 75014 Paris, France \\ 5 DAEC, Observatoire de Paris, Université Paris VII, CNRS (UA 173), 92195 Meudon Cedex, France \\ ${ }^{6}$ Osservatorio Astronomico di Bologna, via Ranzani 1, 40127 Bologna, Italy
}

Received 22 December 2000 / Accepted 18 September 2001

\begin{abstract}
In the present paper, we show that elliptical galaxies (Es) obey a scaling relation between potential energy and mass. Since they are relaxed systems in a post violent-relaxation stage, they are quasi-equilibrium gravitational systems and therefore they also have a quasi-constant specific entropy. Assuming that light traces mass, these two laws imply that in the space defined by the three Sérsic law parameters (intensity $\Sigma_{0}$, scale $a$ and shape $\nu$ ), elliptical galaxies are distributed on two intersecting 2-manifolds: the Entropic Surface and the Energy-Mass Surface. Using a sample of 132 galaxies belonging to three nearby clusters, we have verified that ellipticals indeed follow these laws. This also implies that they are distributed along the intersection line (the Energy-Entropy line), thus they constitute a one-parameter family. These two physical laws (separately or combined), allow to find the theoretical origin of several observed photometrical relations, such as the correlation between absolute magnitude and effective surface brightness, and the fact that ellipticals are located on a surface in the $\left[\log R_{\mathrm{eff}},-2.5 \log \Sigma_{0}, \log \nu\right]$ space. The fact that elliptical galaxies are a one-parameter family has important implications for cosmology and galaxy formation and evolution models. Moreover, the Energy-Entropy line could be used as a distance indicator.
\end{abstract}

Key words. galaxies: clusters: individual (Coma, Abell 85, Abell 496) - galaxies: elliptical and lenticular, cD galaxies: fundamental parameters - distance scale - gravitation

\section{Introduction}

Elliptical galaxies (Es) present a striking regularity in their global luminosity distributions in the sense that, within a wide range of sizes, their light profiles can be described by simple functions, such as the Sérsic law (Caon et al. 1993; Graham \& Colless 1997; Prugniel \& Simien 1997), which is a generalization of the de Vaucouleurs $R^{1 / 4}$ profile. This regularity implies that ellipticals constitute a well defined family of galaxies. The regular properties of Es have been the subject of different approaches, concerning both photometric and spectroscopic parameters, resulting in well known relations such as the FaberJackson and Kormendy relations (Faber \& Jackson 1976; Kormendy 1977) or the Fundamental Plane (Djorgovski \& Davies 1987; Dressler et al. 1987). Es are supposed to be formed under non collisional processes, where dissipation

Send offprint requests to: I. Márquez, e-mail: isabel@iaa.es is expected to be negligible. Under these circumstances, both the initial conditions and the gravitational forces are expected to have a crucial influence on their properties.

In previous papers, we have addressed the question of how the properties of Es may be a consequence of the physical laws they should obey. In Gerbal et al. (1997) and Lima Neto et al. (1999, hereafter Paper I), we have calculated the specific entropy of Es and shown that the results of analysing the cluster Es with Sérsic profiles are compatible with a unique value for this specific entropy, assuming a constant mass/luminosity ratio. In a subsequent paper, Márquez et al. (2000, hereafter Paper II), we have shown that the specific entropy of Es was in fact correlated with the logarithm of the total luminosity while the specific entropy of simulated halos of collisionless particles was correlated with the logarithm of the total mass. Moreover, we suggested that another physical law must be operating in order to explain that Es reside in a thin 
line embedded in a surface defined by a (almost) constant specific entropy.

In this paper, we show that this second physical law is in fact a scaling relation between the potential energy and mass; photometrical relations can be naturally derived from this scaling law under some simplifying hypotheses. We also analyse what the consequences on the origin and evolution of Es are. In Sect. 2 we present a short theoretical introduction to the concepts we will deal with, from which we derive a possible explanation for the existence of an energy-mass relation, and we describe the passage from theoretical parameters to observational ones using the Sérsic profile. We improve our analysis taking into account a possible dependence of the mass to light ratio on the total luminosity. In Sect. 3 we show that similar scaling relations are also found in other types of self-gravitating systems. In Sect. 4 we obtain the relations among the Sérsic parameters and we compare the theoretical predictions with the results from observational data in Sect. 5. The discussion and conclusions are given in Sect. 6.

\section{Theoretical background}

In the standard model of structure formation, initially small perturbations in the cosmic density field start growing in amplitude at the same rate. At a given epoch, when their overdensity relative to the average density is larger than a critical threshold that depends on the assumed cosmology, they stop expanding with the Universe and collapse. Bound objects then settle in an equilibrium configuration through gravitational and radiative processes.

In the following (Sects. 2.1 and 2.2), we propose two laws that elliptical galaxies should obey if they form and reach (quasi) equilibrium under the action of gravitational processes only. As a first approximation, we will assume that radiative processes play a minor role compared to gravitation.

\subsection{Specific entropy}

Elliptical galaxies are thought to be in a quasi-equilibrium state, implying that they should obey the virial theorem. The second law of thermodynamics states that a system in equilibrium is in a maximum entropy configuration. Since elliptical galaxies are gravitational systems, they never really reach an equilibrium state during the time scale of the two-body relaxation. However, even if the entropy $S$ of an E galaxy is ever increasing on a secular time scale, after violent relaxation one may consider that the system is in a quasi-equilibrium stage, which is equivalent to saying that the entropy is quasi-stationary.

Several works have been devoted to the problem of the entropy in gravitational systems (see for instance Merritt 1999, and references therein). In a previous paper (Paper I), we have shown that, assuming that an elliptical galaxy is in a quasi-equilibrium stage, spherically symmetric with an isotropic velocity distribution and constant mass-luminosity ratio, a thermodynamic entropy function can be defined.

In Paper II, instead of using the thermodynamic definition of the entropy, we adopted the microscopic Boltzmann-Gibbs entropy, defined from the distribution function in the 6-dimensional phase space. In order to compare objects of different masses in a consistent way, we have introduced the specific entropy, i.e. the entropy normalized to the mass:

$s \equiv \frac{S}{M}=\frac{-\int f \ln f \mathrm{~d}^{3} x \mathrm{~d}^{3} v}{\int f \mathrm{~d}^{3} x \mathrm{~d}^{3} v}$,

where $f$ is the distribution function. We have then shown that the specific entropy $s$, computed under the previous hypotheses and using the Sérsic profile is almost the same among Es (within 10\%). In fact, based on $N$-body simulations, we suggested that $s$ is not strictly unique but, as a result of merging processes, weakly depends on the logarithm of the galactic mass. We then showed that the specific entropy and the luminosity are tightly correlated. Adopting an appropriate $M / L$ ratio (see Sect. 2.3.1 below) this translates to:

$s=s_{0}+\delta_{s} \ln M$

where $s_{0}$ and $\delta_{s}$ are to be determined by observations.

\subsection{Scaling relation between potential energy and mass}

Let us consider a region with a characteristic length $\lambda_{\mathrm{i}}$ and a mean density $\rho_{\mathrm{i}}$, in the initial density field (all quantities will be indexed with $\mathrm{i}$ - for initial). This region is supposed to be the location of a present E galaxy (its properties will be indexed with $\mathrm{p}$ - for present).

One may suppose, at least to zeroth order, that the following quantities are conserved during the formation and subsequent evolution of the structure:

1. the total mass: $M_{\mathrm{i}}=M_{\mathrm{p}}=M$;

2. the total energy: $E_{\mathrm{i}}=E_{\mathrm{p}}=E$.

The total energy at the initial time is:

$E=T_{\mathrm{i}}+U_{\mathrm{i}} \simeq U_{\mathrm{i}}$

(where $T$ and $U$ are the kinetic and potential energies), because the kinetic energy is negligible compared to the potential energy.

At the present stage, when the galaxy is already relaxed, the two following relations are verified:

$T_{\mathrm{p}}+U_{\mathrm{p}}=E$,

and

$2 T_{\mathrm{p}}+U_{\mathrm{p}}=0$.

Equation (5) is the virial relation, which of course does not hold in the i-case. 
Playing the game of addition, subtraction and comparison, we finally obtain the usual relation for an initially cold gravitational system that collapses and reaches equilibrium:

$U_{\mathrm{p}} \simeq 2 U_{\mathrm{i}}$.

Notice that, if the initial kinetic energy is taken into account, the above relation should read $U_{\mathrm{p}}=2 U_{\mathrm{i}}+2 T_{\mathrm{i}}$.

In the initial linear regime all quantities, such as the typical perturbation length $\lambda_{\mathrm{i}}$, its mass $M$, and its potential energy $U$, are related to one another by scaling relations, e.g. (we will adopt hereafter $G=1$ ):

$M \propto \lambda_{\mathrm{i}}^{3} \quad$ and $\quad U_{\mathrm{i}} \propto M^{2} / \lambda_{\mathrm{i}}$,

implying that:

$U_{\mathrm{i}} \propto \frac{M^{2}}{M^{1 / 3}} \propto M^{5 / 3}$.

Using relation (6), we then obtain:

$U_{\mathrm{p}} \propto M^{5 / 3}$.

If we consider the (small) contribution of the initial kinetic energy then the above relation will be tilted, $U_{\mathrm{p}} \propto$ $M^{\varepsilon+5 / 3}$, where $\varepsilon$ is a small number depending on the exact initial conditions, e.g., the contribution of $T_{\mathrm{i}}$ to the total energy and the initial density fluctuation power spectrum.

Equation (9) can be also written as:

$\ln \left(U_{\mathrm{p}}\right)-5 / 3 \ln (M)=e_{0}$,

where $e_{0}$ is a constant to be determined by observations.

\subsection{From theory to observations}

\subsubsection{Simplifying hypotheses}

The relations given in Eqs. (2) and (10) are both theoretically motivated laws that need to be translated into observable quantities if one wants to test them observationally. In other words, we need to go from the observed two dimensional light distribution to a three dimensional mass distribution. Rather than doing a detailed dynamical and morphological model, we will assume some simplifying hypotheses: spherical symmetry, isotropic velocity dispersion tensor and a mass to light ratio independent of galactic radius $R$.

The last of these hypotheses, i.e., $M / L \equiv \mathcal{F}(R)=$ constant, implies that the mass distribution follows the light profile, with the same scale length and shape. Notice that the above "constant" may in fact depend on the total galaxy mass or luminosity (as we will see below). This hypothesis is clearly a rough simplification: X-ray observation of giant ellipticals (e.g. Loewenstein \& White 1999), gravitational lensing analyses (Griffiths et al. 1998), and dynamical studies (e.g. Gerhard et al. 2001), suggest that $\mathcal{F}(R)$ increases with radius. This increase, however, seems to be important only beyond $R \gtrsim 2 R_{\text {eff }}$ and current data is compatible with $\mathcal{F}(R) \approx$ constant for $R \lesssim 2 R_{\text {eff }}$ (Kronawitter et al. 2000).

It has been argued (Bertin et al. 1992) that a significant amount of dark matter can coexist with the stellar component without distorting the luminosity profile either if both the stellar and dark matter profiles have the same shape or if the dark matter is much more diffuse, i.e., with a larger scale length and lower central density. The former case corresponds to our hypothesis of a constant $\mathcal{F}(R)$. In the latter case, the contribution of the dark matter component will be important only in the external region of the galaxy.

The spherical symmetry and isotropy hypotheses imply that the distribution function is simply a function of one variable, the binding energy. Although these hypotheses may hold in the internal region, they must be poor approximations in the external regions of the most flattened galaxies. However, there is no clear correlation between ellipticity and luminosity and the effect of neglecting the flattening should not produce any systematic error.

Finally, we will assume that mass and luminosity are related by a power-law:

$\mathcal{F}(L)=k_{F} L^{\alpha}$,

where the index $\alpha$ may be zero (equal mass to light ratio for all ellipticals). The above functional form is suggested, e.g., by the analysis of the tilt of the Fundamental Plane assuming homology (e.g., Burstein et al. 1997; Jørgensen et al. 1999), where $\alpha \approx 0.22-0.32$. Notice that the hierarchical merger simulations discussed by Capelato et al. $(1995,1997)$ give a natural explanation for the origin of the Fundamental Plane under the hypothesis of a constant value of $\mathcal{F}$.

\subsubsection{Working model}

With the above hypotheses, the mass, potential energy and specific entropy are easy to compute from the light distribution.

Our working model is the Sérsic (1968) luminosity profile:

$\Sigma=\Sigma_{0} \exp \left[-(R / a)^{\nu}\right]$

which describes well the observed surface profile distribution of ellipticals (e.g., Caon et al. 1993; Graham \& Colless 1997; Prugniel \& Simien 1997, see also Sect. 6.1), and allows to compute analytically good approximations of all the needed quantities. We therefore adopt this profile to model the observed surface brightness distribution of a sample of elliptical galaxies (see Sect. 5.1), and to compute the physical quantities of interest, like the total mass, the potential energy, the specific entropy, etc. (see Tables A.1 and A.2 in the Appendix for a summary of the formulae we use).

Note that the Sérsic law is a non-homologous generalization of the de Vaucouleurs profile, in the sense that a third parameter describing the shape of the distribution 
(the structural parameter $\nu$ ) is introduced and left free, instead of being fixed to 0.25 . This of course allows a better fit to the observations (Graham \& Colless 1997, and Papers I and II).

We assume that only one dynamical component describes an elliptical galaxy and ignore the possible presence of nuclear cusps or embedded discs. This simplification is motivated by the fact that these components, if present, usually represent at most a few percent of the total galactic mass (cf., de Zeeuw \& Franx 1991; Kormendy \& Richstone 1995, and references therein; see also Saglia et al. 1997).

We stress here that we are doing a pure photometrical analysis and therefore such a working model is required (with all its simplifying hypotheses) so that we are able to derive dynamically related quantities like the specific entropy or the potential energy.

\section{Scaling relations: Elliptical galaxies only?}

The derivation of the scaling relation between potential energy and mass is based on fundamental principles (mass and energy conservation). It can therefore be applied to any object formed by the growth of density perturbations, driven by gravitational interaction. Dark matter (DM) halos are therefore expected to follow the same relation.

Assuming that collisionless $N$-body simulations provide a suitable description of DM halo formation, relation (10) should then also be found automatically in the results of such simulations. We have tested this hypothesis by considering a cosmological $N$-body simulation with $512^{3}$ particles in a $479 \mathrm{Mpc} / h$ side box, characterized by the following parameters: a $\Lambda$ CMD cosmological model, with density parameters $\Omega_{0}=0.3$ and $\Omega_{\Lambda}=0.7$, Hubble constant $H=100 \mathrm{hm} \mathrm{s}^{-1} \mathrm{Mpc}^{-1}, h=0.7$, and normalization $\sigma_{8}=0.9$ (Yoshida et al. 2001). The corresponding particle mass is $6.8 \times 10^{10} h^{-1} M_{\odot}$. A sphere of radius $r=7 h^{-1} \mathrm{Mpc}$ around a very massive DM halo $(M \simeq$ $2.3 \times 10^{15} h^{-1} M_{\odot}$, virial radius $R_{\mathrm{vir}} \simeq 2.7 h^{-1} \mathrm{Mpc}$ ) has been selected in the original simulation, and re-simulated at higher resolution (a factor $\sim 35$ increase, to get a mass particle of about $\left.2 \times 10^{9} h^{-1} M_{\odot}\right)$. Assuming a minimum mass of 10 particles per halo, this allows to resolve halos down to $2 \times 10^{10} h^{-1} M_{\odot}$.

Figure 1 shows the distribution of potential energy versus mass for the halos found within the selected sphere, their masses ranging from $10^{12}$ to $10^{15} h^{-1} M_{\odot}$. We have selected the halos that had their kinetic to potential energy ratio closer to the virial theorem value, i.e., the ones satisfying $0.8 \leq 2 T /|U| \leq 1.2$. Due to the high resolution of the simulation, the computation of the potential energy is very accurate, at least for all halos more massive than $\sim 10^{11} M_{\odot}$, i.e. composed by more than 100 particles.

A fit to the data indicates a power-law index of $1.69 \pm 0.02$, very close to the theoretical value of $5 / 3$. The difference may be due to the fact that the $N$-body simulation takes into account all dynamical processes (mass and energy exchange and/or loss) self-consistently.

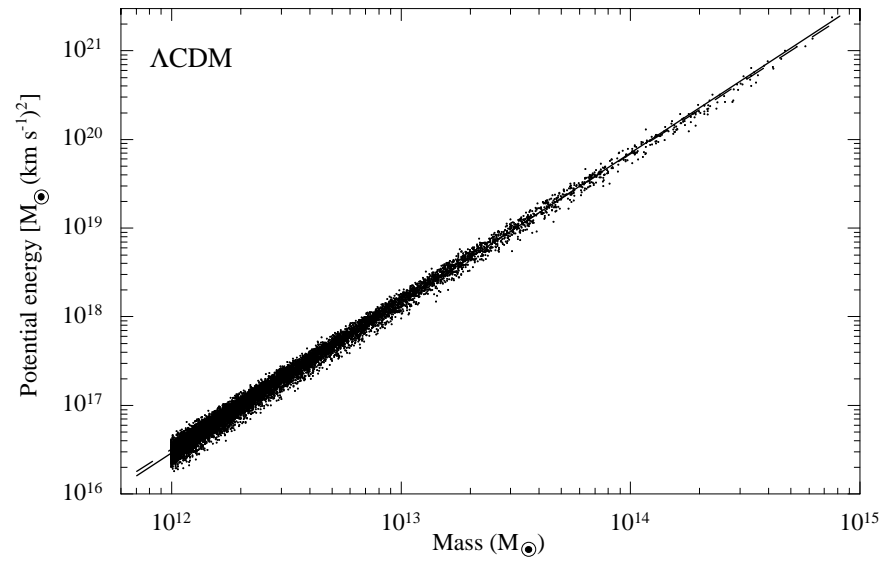

Fig. 1. Potential energy-mass relation for about 29000 dark matter halos selected in a high-resolution $N$-body simulation (see text). The solid line is the best fit to the data, with a slope of $1.69 \pm 0.02$, in very good agreement with the expected theoretical value of $5 / 3$ (dashed line).

We have also verified that this relation is independent on cosmology. In fact, we found the same slope of the potential-energy mass relation for DM halos in all the GIF Simulations (Kauffmann et al. 1999), run for four different cosmological models: SCDM, OCDM, $\Lambda \mathrm{CDM}, \tau \mathrm{CDM}$.

A comparable simulation of dark matter haloes was done by Jang-Condell \& Hernquist (2001) in a much lower mass range. In this simulation, each particle represents $4.6 \times 10^{4} M_{\odot}$, and the range of halo masses is $4 \times 10^{5} M_{\odot}$ to $4 \times 10^{8} M_{\odot}$. The potential energy calculated for 2501 bound halos also seems to follow a power law $|U| \propto M^{5 / 3}$.

This is an example of the universality of the scaling relation between potential energy and mass. Whereas out of the scope of this paper, numerous questions obviously arise, such as the dependence (or independence) of the index with redshift or with the cosmology assumed in the simulation.

Interestingly, the analysis of ROSAT-PSPC images of 24 clusters has also revealed the existence of a scaling law between the cluster potential energy and the mass of the X-ray gas, with a dependence $U \propto M^{1.72 \pm 0.05}$, again close to $5 / 3$ (see Durret et al. 2001). Such a scaling law therefore appears to be general for self-gravitating systems.

\section{Predicted relations among the Sérsic parameters}

We now show that the two relations introduced in Sects. 2.1 and 2.2, constraining the distribution of gravitational matter in galaxies, define two surfaces intersecting each other in the Sérsic parameter space $\left[\nu, a, \Sigma_{0}\right]$.

The first relation comes from the relation between the specific entropy, $s$, and the mass, $M$, that can be expressed as

$s=s_{0}+\delta_{s} \ln M=s_{0}+\delta_{s} \ln (\mathcal{F} L)$, 


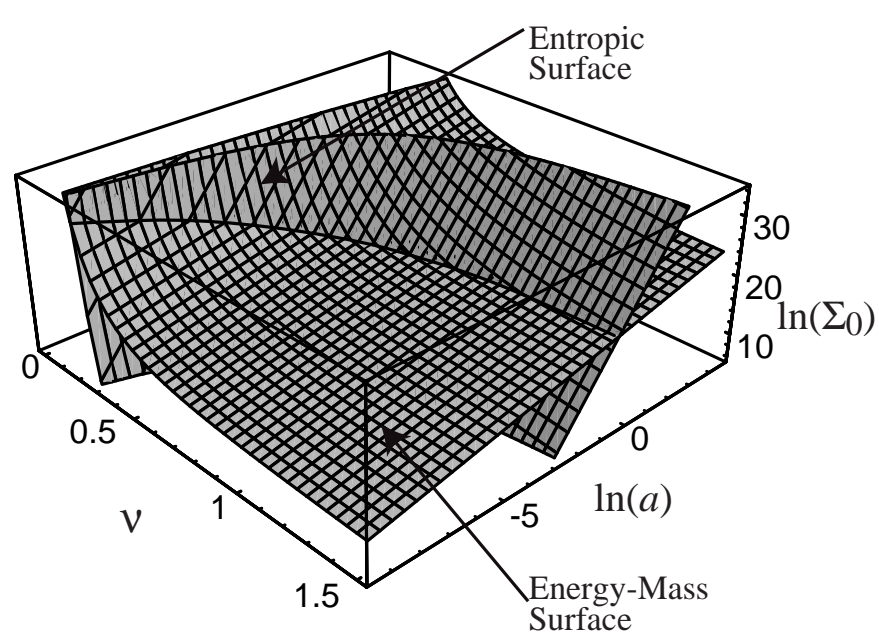

Fig. 2. 3-D representation of the specific entropy and energymass 2-manifolds, using the coordinates: $\left[\log \Sigma_{0}, \log a, \nu\right]$. In this example, we assume $M \propto L$.

cf. Eq. $(2)^{1}$. From the expression of the entropy in Table A.1, it is straightforward to obtain the Entropic Surface in terms of the Sérsic parameters, Eq. (A.1) shown in the Appendix.

The second relation is obtained using the definition of potential energy and mass given in Tables A.1 and A.2, that can be cast in a general form:

$U=k_{U} M^{\beta}$ or, equivalently, $\ln U=e_{0}+\beta \ln (\mathcal{F} L)$,

where $e_{0} \equiv \ln k_{U}$ and we have dropped the index "p". Using the luminosity and the potential energy derived for a mass distribution following the Sérsic profile (cf. Table A.1), it is easy to obtain the Energy-Mass Surface in terms of the observed photometric Sérsic parameters. The formula describing this surface is shown in the Appendix, Eq. (A.2).

A three dimensional representation of these two surfaces is given in Fig. 2, in the $\left[\nu, a, \Sigma_{0}\right]$ space. The intersection line (called the Entropy-Energy line in Paper II) is the locus on which elliptical galaxies are distributed in the space of the Sérsic parameters.

Combining relations (A.1) and (A.2), i.e. calculating the intersection of the Entropic and Energy-Mass surfaces, two-by-two relations between the Sérsic parameters can be obtained. The general $[a, \nu]$ and $\left[\Sigma_{0}, \nu\right]$ relations are given in the Appendix. For the particular case where $\alpha=0$ (constant $M / L$ ratio), $\beta=5 / 3$ (cf. Sect. 2.2 ) and $\delta_{s}=0$ (unique specific entropy), we have for the $[a, \nu]$ relation:

$\ln (a)+F_{a}(\nu)=-\frac{3}{4} e_{0}+\frac{1}{6} s_{0}+\frac{3}{4} \ln G$,

${ }^{1}$ In Paper II, the specific entropy is defined with a different normalization: $s^{\prime}=-\frac{1}{M} \int \mathrm{d} x^{3} \mathrm{~d} v^{3} f \ln f$, where $\int \mathrm{d} x^{3} \mathrm{~d} v^{3} f=$ $M$. Here, we use the normalization $\int \mathrm{d} x^{3} \mathrm{~d} v^{3} f^{*}=1$ and compute the specific entropy $s$ using the normalized distribution function $f^{*}$. With simple algebra it can be shown that $s=s^{\prime}+\ln M$.

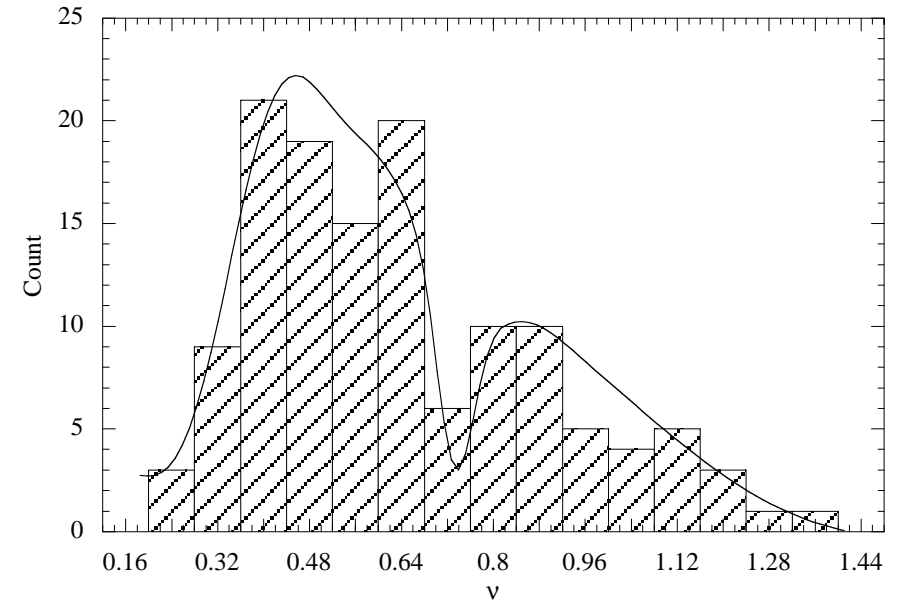

Fig. 3. Histogram of the $\nu$ distribution superimposed on the wavelet reconstruction of the density distribution.

with $F_{a}(\nu)=\frac{3}{4} \ln R_{g}^{*}(\nu)-\frac{1}{4} \ln M_{2}^{*}(\nu)+\frac{1}{6} F_{2}(\nu)$,

and, for the $\left[\Sigma_{0}, \nu\right]$ relation:

$\ln \left(\Sigma_{0}\right)+F_{\Sigma}(\nu)=\frac{9}{4} e_{0}+\frac{1}{6} s_{0}-\frac{9}{4} \ln G$,

with $F_{\Sigma}(\nu)=-\frac{9}{4} \ln R_{g}^{*}(\nu)+\frac{3}{4} \ln M_{2}^{*}(\nu)+\frac{1}{6} F_{2}(\nu)$.

The relation between $a$ and $\Sigma_{0}$ is obtained by combining relations (15) and (16), with $\nu$ as a parameter.

\section{Predictions faced to observations}

\subsection{Data set and distribution of the $\nu$ parameter}

The sample of elliptical galaxies we consider is described in detail in Paper II. It is composed of 68 galaxies in the Coma Cluster, 30 in Abell 85 and 34 in Abell 496; their cluster membership is confirmed by their redshifts.

For all these galaxies, we have fitted the observed surface brightness distribution (obtained by CCD imaging in the $V$ band) by means of the Sérsic integrated profile, i.e., using the growth curve fitting method (see Papers I and II, for a detailed description and discussion of the fitting procedure). As explained in our previous papers, S0 galaxies were excluded from our sample because they fall out of the correlations between the Sérsic parameters. To be able to use all the data as a single set, the fitting parameters $a$ and $\Sigma_{0}$ have been expressed in physical units, i.e., in $\mathrm{kpc}$ and $L_{\odot} / \mathrm{kpc}^{2}$ respectively ${ }^{2}$.

While in the de Vaucouleurs law a single value of $\nu$ is used $(\nu=1 / 4)$, our data fitting reveals that this parameter can cover a range of values, which are not centered on 0.25. We give in Fig. 3 the density distribution of $\nu$ obtained by wavelet reconstruction (see Fadda et al. 1998 for a description of the method). The distribution is bi-modal, with a first maximum around $\nu=0.44$ and a second, less pronounced, one around $\nu=0.82$ (close to 1 , the typical value for dwarf spheroidal galaxies); notice that in the

\footnotetext{
${ }^{2}$ We have used $H_{0}=100 \mathrm{~km} \mathrm{~s}^{-1} \mathrm{Mpc}^{-1}$ and $q_{0}=1 / 2$.
} 


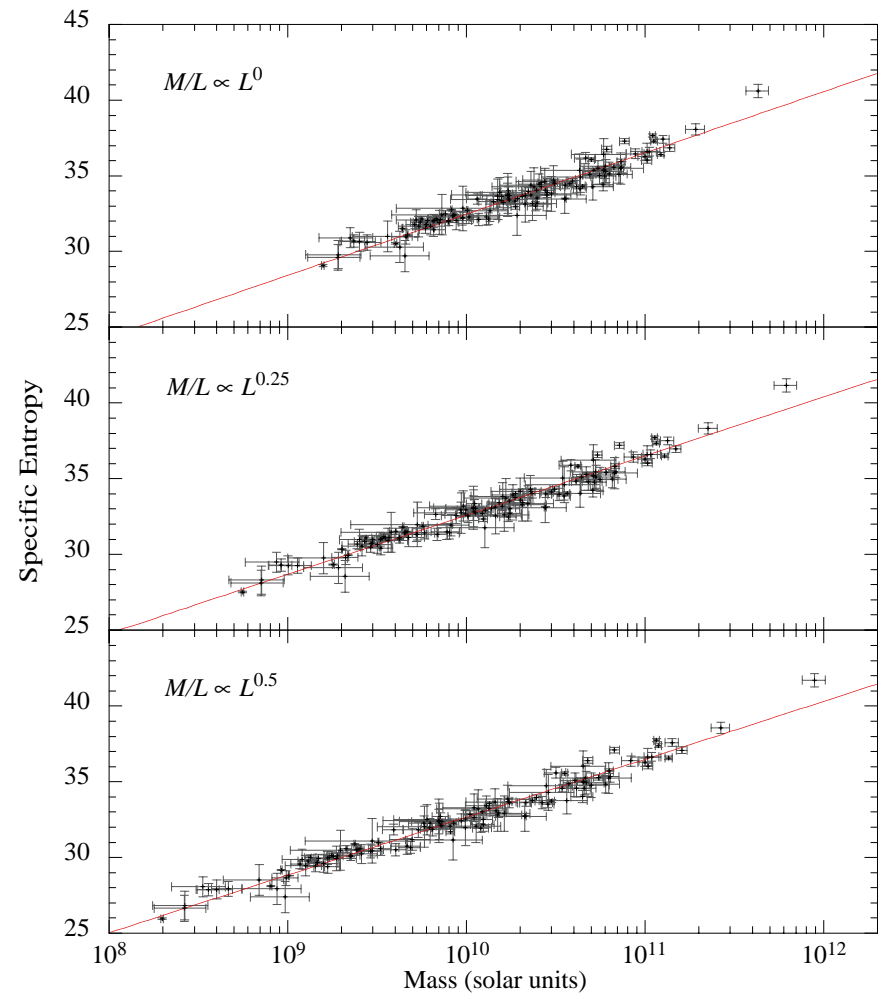

Fig. 4. Specific entropy versus mass for three values of $\alpha$. The bisector-OSL fits to our 132 galaxy sample are shown as full lines.

previously published $\nu$-histogram (Paper I), this second maximum is already present, although not as significantly. This is due to the fact that we are now working with a larger sample, resulting from the combination of three separate data sets. It is worth noting however that our sample is more than $97 \%$ complete only up to magnitude $V \sim 20$ and the giant ellipticals of Coma were not included because of saturation of the CCD; besides, the CCD fields do not cover the entire clusters, but only the central parts (in particular for Abell 85 and 496). Therefore both the position of the two peaks and their relative intensities may be somewhat different when considering complete samples.

\subsection{Entropy and energy-mass relations}

As discussed in Sect. 2.3, the mass, entropy and potential energy of all the galaxies in our sample were computed under the hypothesis that light traces mass.

The uniqueness of the specific entropy of Es has been discussed and established in Paper I, at least at zeroth order. In Paper II we have shown that, as a result of energy and mass exchange in merging processes, $s_{0}$ weakly depends on the mass. A constant value for the mass-to-light ratio was assumed in these two papers.

We now calculate the specific entropy as a function of mass for three mass to light relations: $M / L \propto L^{0}$ (constant mass to light ratio), $L^{0.25}$ (from Fundamental Plane studies, e.g., Burstein et al. 1997) and $L^{0.5}$ (extreme case).

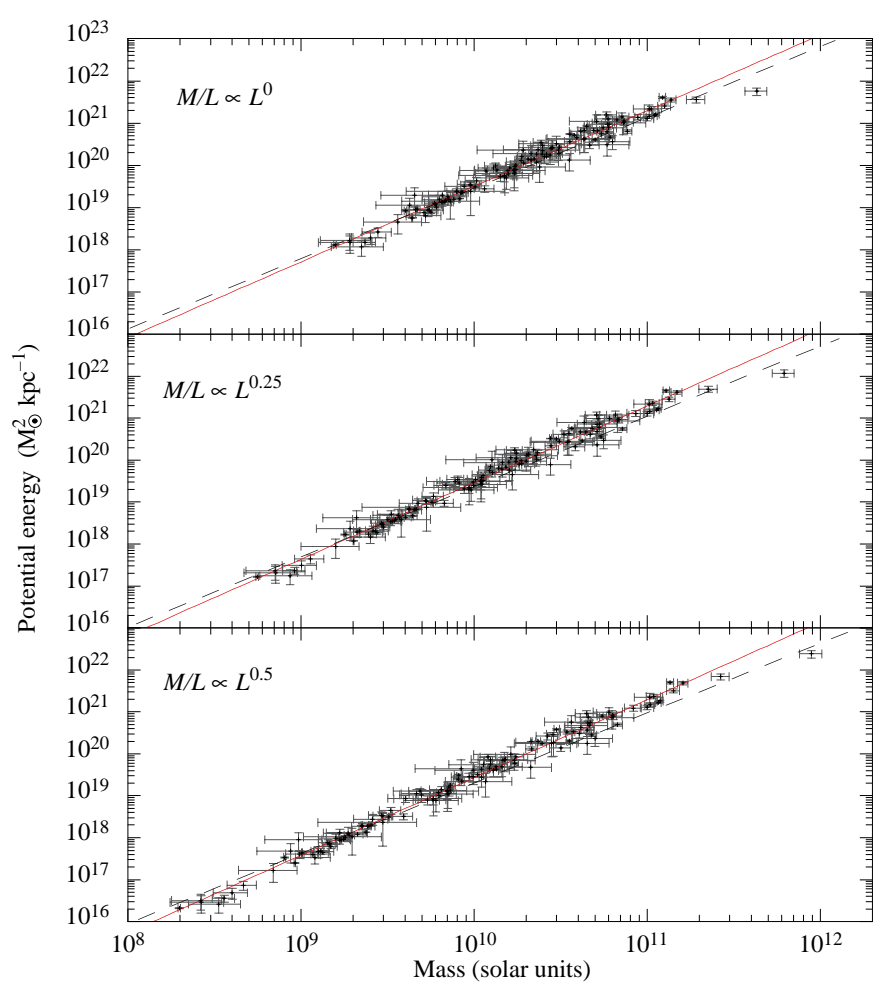

Fig. 5. Potential energy (with $G=1$ ) versus mass for three values of $\alpha$. The power-law fits (full lines) give slopes of 1.79, 1.83 and 1.85 respectively from top to bottom (see Table 1 ). The theoretical relation, with an index of $5 / 3$, is also shown (dashed lines).

The results are shown in Fig. 4 and the best power law fits corresponding to Eqs. (13) and (14) are given in Table 1.

The potential energy is displayed in Fig. 5 as a function of total mass (adopting $G=1$ ). A bisector-OLS (Ordinary Least Square) power law fit (Feigelson \& Babu 1992) gives a indexes $\beta=1.79,1.83$ and 1.85 for $M / L \propto L^{0}, L^{0.25}$ and $L^{0.5}$ respectively (see Table 1 ). However, if we impose $\beta=5 / 3$ (as predicted in Sect. 2.2, see Eq. (9)), the fit is still good enough (within $2 \sigma$, cf. Table 1 ) to confirm that Eq. (10) is a good approximation. Fish (1964) analysed a set of 24 Es, fitting their surface brightness profiles with the de Vaucouleurs law, and found a power law index $\beta=$ $3 / 2$. The improved accuracy of both our data and our fitting procedure (using the Sérsic profile), as well as our larger sample, allow us to definitely exclude $\beta=3 / 2$ (see also Sect. 3).

\subsection{Correlations between the Sérsic profile parameters}

From a mathematical point of view the primary parameters $a, \Sigma_{0}$ and $\nu$ entering in the definition of the Sérsic profile are independent from each other. But as discussed in Sect. 4, the entropy and energy-mass relations, Eqs. (A.1) and (A.2), imply the existence of two-by-two relations between the three Sérsic parameters. From the observational point of view, we know that $a, \Sigma_{0}$ and $\nu$ are in fact correlated (Young \& Currie 1995; Gerbal et al. 1997). We now 


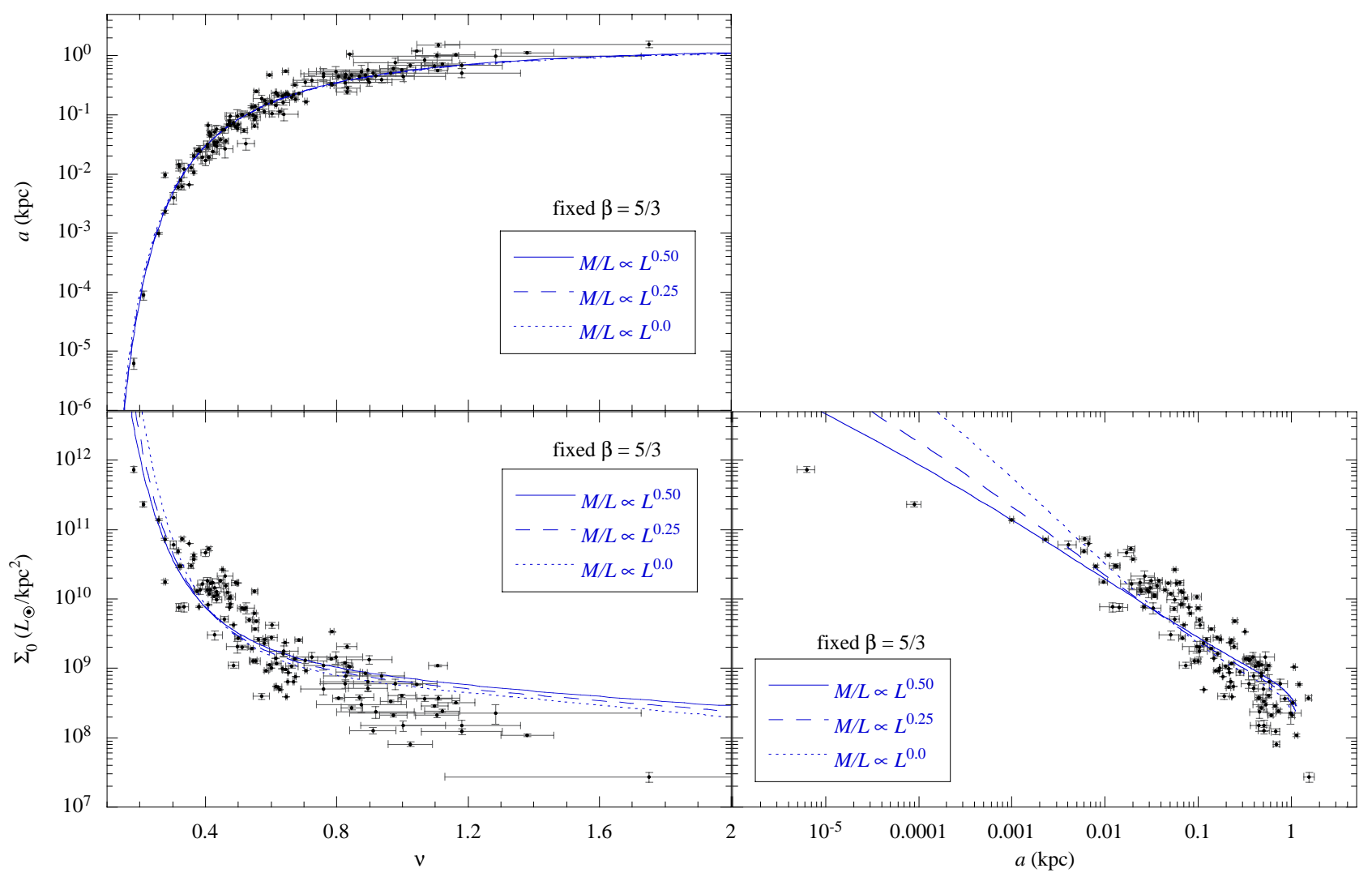

Fig. 6. Pairwise correlation between the Sérsic parameters $[a, \nu],\left[\Sigma_{0}, \nu\right]$ and $\left[\Sigma_{0}, a\right]$ (counter clockwise from top left). The lines superimposed in each panel are the correlations predicted from the theoretical slope $\beta=5 / 3$ for $\alpha=0.0,0.25$ and 0.50 .

Table 1. Fitting results for the specific entropy and potential energy scaling relations. The slope of the mass-luminosity ratio is $\alpha, s_{0}$ and $\delta_{s}$ are the parameters of the entropy-mass relation; $e_{0}$ and $\beta$ are energy-mass parameters.

\begin{tabular}{ccccc}
\hline \multicolumn{1}{c}{$\alpha$} & $s_{0}$ & $\delta_{s}$ & $e_{0}$ & $\beta$ \\
\hline 0.0 & $-8.0 \pm 1.2$ & $1.76 \pm 0.06$ & $3.68 \pm 0.03$ & $1.79 \pm 0.06$ \\
0.25 & $-6.5 \pm 1.0$ & $1.70 \pm 0.04$ & $2.74 \pm 0.05$ & $1.83 \pm 0.06$ \\
0.50 & $-5.5 \pm 0.8$ & $1.66 \pm 0.03$ & $2.08 \pm 0.06$ & $1.85 \pm 0.06$ \\
\hline
\end{tabular}

Notice that the values of $s_{0}$ for $\alpha=0$ are different from the ones shown in Paper II due to our different definition of the specific entropy (cf. Sect. 4 and Table A.1).

explain these correlations as originating from the physical laws discussed in Sect. 2.

Figures 6 and 7 show the $[a, \nu],\left[\Sigma_{0}, \nu\right]$, and $\left[\mathrm{a}, \Sigma_{0}\right]$ relations for our sample of galaxies. The corresponding theoretical relations, computed for $\alpha=0.0,0.25,0.50$ are superimposed on the data for two cases: the fixed value of $\beta=5 / 3$ (the theoretical prediction as in Eq. (8)) and the values of $\beta$ given in Table 1 . The theoretical prediction lies close to the data points, in particular for big galaxies (those with the smallest $\nu$ for $\beta=5 / 3$ - see Sect. 6.1). We note that in both cases the effect of changing the $M / L$ ratios is small.

\subsection{From theoretical to observed correlations}

The well known photometric correlations which characterize elliptical galaxies, are usually expressed by means of astrophysical quantities like $R_{\text {eff }},\langle\mu\rangle_{\text {eff }}$, and/or the absolute magnitude of the galaxies. Since these quantities are combinations of the primary parameters defining the Sérsic law, if these observed photometric correlations do indeed contain information on the physics of the objects or on the processes that drove their formation and evolution, one should, in principle, be able to derive them from the "theoretical" relations, Eqs. (2) and (10). In doing so, we show that it is indeed possible to give a physical interpretation to some of the observed correlations and that they are not just artefacts of the definitions of the parameters.

Before going through the comparison between theory and observation however, it is necessary to take into account the following points:

1. The correlations proposed in the literature (e.g., Young \& Currie 1994; Binggeli \& Jerjen 1998) result from fitting procedures; the coefficients are just empirical. Moreover, for the sake of simplicity, attempts are usually done to linearize the correlations. On the contrary, the coefficients that we derive are the consequence of physical laws, and the relations we have found are non-linear.

2. The intersection of the specific entropy and of the energy-mass surfaces is a line that we call the 


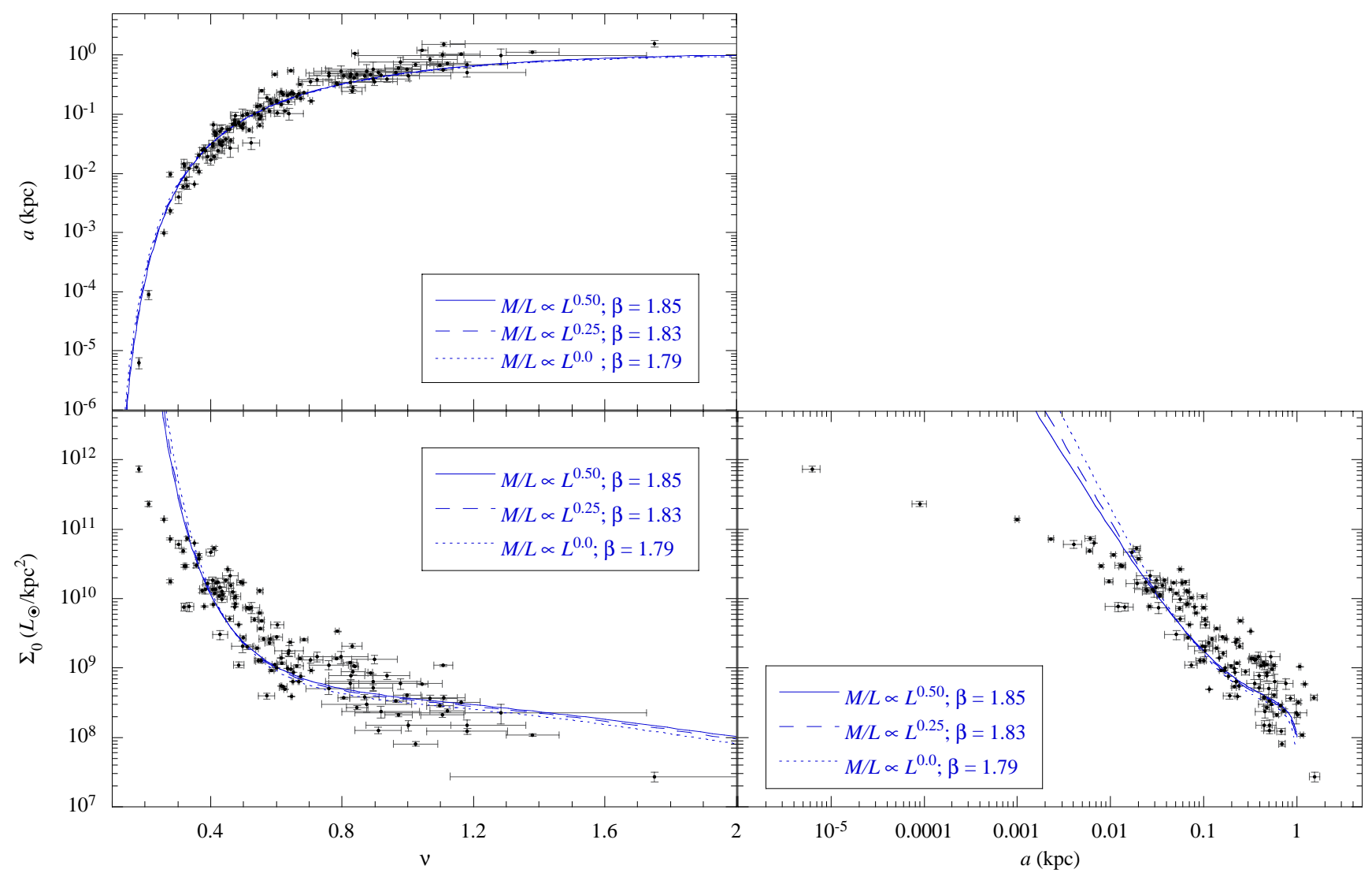

Fig. 7. Pairwise correlation between the Sérsic parameters $[a, \nu],\left[\Sigma_{0}, \nu\right]$ and $\left[\Sigma_{0}, a\right]$ (counter clockwise from top left). The lines superimposed in each panel are the correlations predicted from the Entropic Surface (with $\alpha=0.0,0.25$ and 0.50 ) and the fitted $\beta$ values for the energy-mass scaling relations for these three values of $\alpha$ (see Table 1).

"Energy-Entropy line". The region of this line populated by ellipticals is not coplanar, but can be considered as located in a plane in a restricted range of values.

\subsubsection{Luminosity-effective radius relation}

From the intersection of the specific entropy and of the energy-mass surfaces, Eqs. (A.3) and (A.4) parametrized by $\nu$, we can derive photometrical relations for Es galaxies using the formulae in Table A.2.

In Fig. 8 we plot the total luminosity as a function of $R_{\text {eff }}$ for our data.

Using the definition of the potential energy, relation (10) can be written as:

$e_{0}=\frac{1}{3}\left[\ln M-3 \ln r_{\mathrm{g}}\right]$

where $r_{\mathrm{g}}$ is the gravitational radius and $M$ the mass. Assuming that $R_{\text {eff }}$ is proportional to $r_{\mathrm{g}}$ (which is a very good approximation for galaxies described by the Sérsic profile, see Table A.2), we then have:

$\log L=3 \log R_{\text {eff }}+3 \log k-\log \mathcal{F}+1.30288 e_{0}$,

where $\mathcal{F}$ is the $M / L$ ratio and $k$ is the proportionality constant between $R_{\text {eff }}$ and $r_{\mathrm{g}}$. This relation is drawn with the dot-dashed line in Fig. 8. Notice that the theoretical slope of the $L-R_{\text {eff }}$ relation, in this case, depends only on the slope of the scaling relation between mass and potential energy.

We can relax some simplifying hypotheses, proportionality between $R_{\text {eff }}$ and $r_{\mathrm{g}}$ and constant $M / L$ ratio and obtain a more general relation between $L$ and $R_{\text {eff }}-$ the price to pay is a much more complicate expression, instead of Eq. (18). The theoretical relations obtained for a fixed $\beta=5 / 3$ (left) and for the fitted values of $\beta$ (right) are superimposed for the three different values of $\alpha$. Within the error bars, the relations are compatible with the data. The different curves vary with $M / L$ when $\beta$ is fixed $(\beta=5 / 3)$ but lead to similar curves when $\beta$ is free.

Our data and theoretical prediction are steeper than the results found in the literature. Binggeli et al. (1984) derive $L \propto R_{\text {eff }}^{2}$ for galaxies with $0.2 \lesssim R_{\text {eff }} \lesssim 5 \mathrm{kpc}$. For smaller galaxies, however, the slope steeps and reaches our theoretical value of 3 . Schombert (1987) also finds that the $L-R_{\text {eff }}$ relation is shallower for the brightest galaxies; for normal ellipticals he finds $L \propto R_{\text {eff }}^{1.8}$. Even though these low values are not incompatible with our data (within the error bars), it should be stressed that previous $L-R_{\text {eff }}$ studies were based on the de Vaucouleurs profile, while we fit our galaxies with the Sérsic law. As we will see below, different modelling (i.e., de Vaucouleurs or Sérsic laws) of Es may change the observed correlations among their global parameters. 


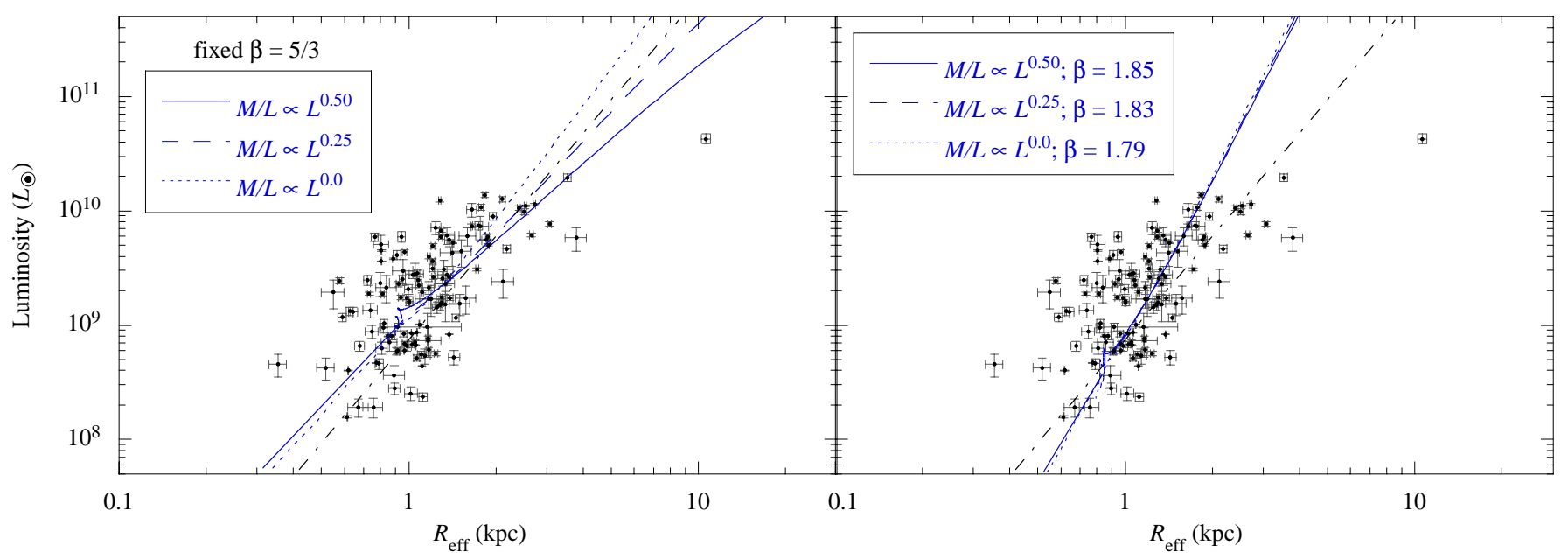

Fig. 8. Luminosity versus effective radius for our 132 cluster ellipticals. The theoretical relations obtained for a fixed $\beta=5 / 3$ (left) and for the fitted values of $\beta$ (right) are superimposed for the three different values of $\alpha$. The theoretical relation given by Eq. (18) is superimposed as a dot-dashed line. The continuous, dashed and dotted lines are obtained using the intersection of the specific entropy-mass and energy-mass relations, Eqs. (A.1) and (A.2).

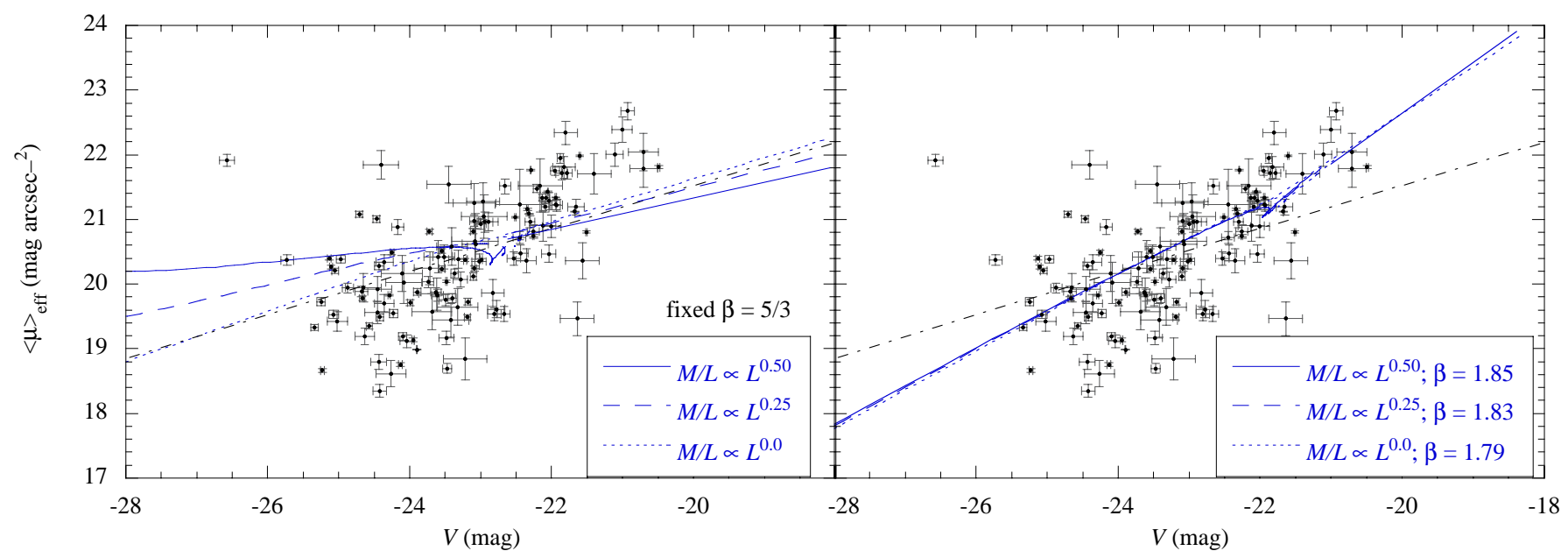

Fig. 9. Mean effective magnitude versus absolute magnitude. The results for the different values of $\alpha$ are plotted for the theoretical prediction $\beta=5 / 3$ (left) and for the fitted values of $\beta$ (right). The theoretical relation given by Eq. (20) is superimposed as a dot-dashed line. The continuous, dashed and dotted lines have the same meaning as in Fig. 8.

\subsubsection{Magnitude-mean surface brightness correlation}

Taking the definition of $\langle\mu\rangle_{\text {eff }}$ :

$\langle\mu\rangle_{\mathrm{eff}}=-2.5 \log L+5 \log R_{\mathrm{eff}}+2.5 \log (2 \pi)$,

we can combine it with the energy-mass scaling relation, Eq. (17), eliminating the effective radius. The results for the different values of $\alpha$ are plotted in Fig. 9. The theoretical relations obtained for a fixed $\beta=5 / 3$ (left) and for the fitted values of $\beta$ (right) are superimposed for the three different values of $\alpha$. The different curves vary with $M / L$ when $\beta$ is fixed $(\beta=5 / 3)$ but lead to similar curves when $\beta$ is free.

If we make the same assumptions as in Sect. 5.4.1 above, we obtain a relation between $\langle\mu\rangle_{\mathrm{eff}}$ and $L$, or equivalently, in terms of the absolute magnitude $(\mathcal{M} \equiv$ $-2.5 \log L)$ :

$$
\langle\mu\rangle_{\mathrm{eff}}=\frac{1}{3} \mathcal{M}-5 \log k+\frac{5}{3} \log \mathcal{F}-2.1715 e_{0}+\frac{5}{2} \log (2 \pi),
$$

traced as a dot-dashed line in Fig. 9, where the variables have the same meaning as in Eq. (18). Note that this relation does not predict two regimes but a single one. This is indeed in agreement with the observations when the data are analysed with the Sérsic law, as done by Jerjen \& Binggeli (1997), and as shown in Fig. 9 for our galaxies. Quantities like $R_{\text {eff }}$ or $\langle\mu\rangle_{\text {eff }}$ are different when obtained through a de Vaucouleurs or a Sérsic fit (Graham \& Colless 1997; Jerjen \& Binggeli 1997): this probably explains why Binggeli et al. (1984), who used the de Vaucouleurs profile, obtain a broken power-law $\langle\mu\rangle_{\mathrm{eff}^{-}}$ $\mathcal{M}$ relation, while Jerjen \& Binggeli (1997) and us, using the Sérsic profile, find a single power-law. 


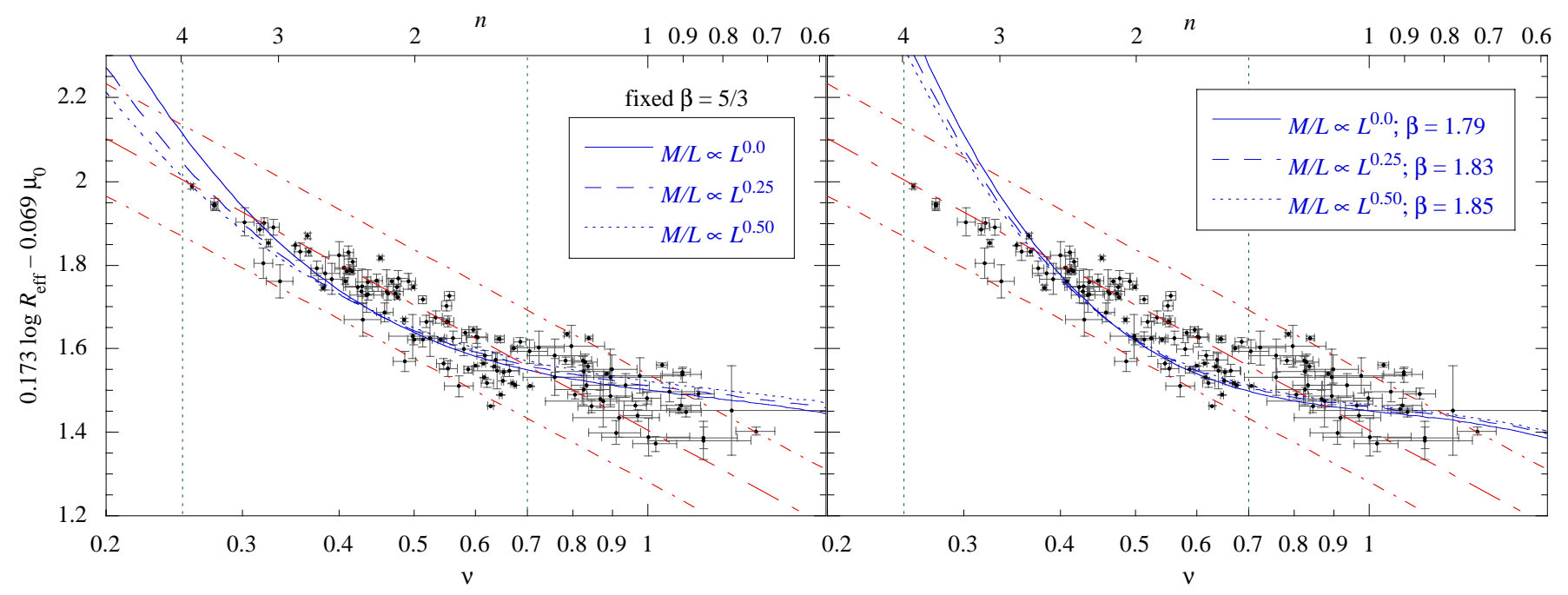

Fig. 10. The theoretical relation given by the generalized form of the right-hand side of Eq. (24) (solid, dashed and dotted lines) is superimposed on the fit (long-short dashed line) obtained by Khosroshahi (2000) (right-hand side of Eq. (21)). The two vertical dotted lines correspond the range in $\nu$ used by Khosroshahi (2000). The dash-dotted lines correspond to $8 \%$ errors on the fit. The shape parameter, $n \equiv 1 / \nu$, is given at the top of the figure to allow direct comparison with other authors.

\subsubsection{Photometric plane}

Khosroshahi et al. (2000) have fitted a set of infra-red profiles of $\mathrm{E}$ and spiral bulges with the Sérsic law. They have shown that these galaxies lie on a plane in the space defined by the set of coordinates $\left[\log R_{\text {eff }}, \mu_{0}, \log \nu\right]$, with $\mu_{0}=-2.5 \log \Sigma_{0}$. Notice that these authors use $n=1 / \nu$ in the Sérsic law. The equation they find for this plane is:

$$
\begin{aligned}
(0.173 \pm 0.025) \log R_{\text {eff }} & -(0.069 \pm 0.007) \mu_{0}= \\
& -\log \nu-(1.18 \pm 0.05) .
\end{aligned}
$$

We will now derive a similar equation from a theoretical point of view. For illustration purpose, we derive here the simplest case with constant mass to light ratio $(\alpha=0)$ and $\beta=5 / 3$. The general case is of course similar but more cumbersome. Including the definition of $\mu_{0}$ and the relation between $R_{\text {eff }}$ and $a$ into Eqs. (A.1) and (A.2), after some straightforward algebra, we obtain two equations:

$\log R_{\mathrm{eff}}-\frac{2}{15} \mu_{0}=\log R_{\mathrm{eff}}^{*}(\nu)+\frac{2}{9} \log e\left(s_{0}-F_{2}(\nu)\right)$,

and

$$
\begin{aligned}
\log R_{\text {eff }}+\frac{2}{5} \mu_{0}= & \log M_{2}^{*}(\nu)-1.94 \log R_{\text {eff }}^{*}(\nu) \\
& -1.303 e_{0}-1.5113 .
\end{aligned}
$$

A linear combination between Eqs. (22) and (23) gives:

$0.173 \log R_{\text {eff }}-0.069 \mu_{0}=K(\nu)$,

with:

$$
\begin{aligned}
K(\nu)= & -0.086125 \log M_{2}^{*}(\nu)+0.4262 \log R_{\mathrm{eff}}^{*}(\nu) \\
& +0.1122 e_{0}+0.025\left(s_{0}-F_{2}(\nu)\right)+0.13016 .
\end{aligned}
$$

Relation (24) is obtained from theoretical relations; on the other hand, relation (21) is a fit to the observed data.
The only differences between these two relations are the two right-hand sides, i.e. $(-\log \nu)$ should be compared to $K(\nu)$. Only the forms of the two functions are to be compared since the constant depends on the units chosen. In Fig. 10, we call attention to the interval [0.25-0.7] in $\nu$, which 2000 have used to define their photometrical plane. In particular, their relation given by Eq. (21) and ours [Eq. (24)] agree within $8 \%$ in this range. The good agreement between the observed points of Khosroshahi et al. and our theoretical relation shows that the photometric plane may be understood as a consequence of the two laws discussed in the present paper. Note however that it is possible to define a "plane" only because a limited range of values has been considered for $\nu$ (see the beginning of Sect. 5.4).

\section{Discussion and conclusions}

\subsection{Ellipticals as a single parameter family}

The Sérsic profile is certainly not the ultimate profile to reproduce the surface brightness of ellipticals, from brighter galaxies to dwarf ellipticals (see Jerjen \& Binggeli 1997). The Sérsic profile cannot apply to fine features such as boxy isophotes, central black hole, etc., but it allows a fit of sufficient quality for our aims, since we use it only to describe large scale properties (Caon et al. 1993; Graham \& Colless 1997; Prugniel \& Simien 1997; Márquez et al. 2000). A finer description, which is out of the scope of this paper, would certainly require another parameter to reproduce the whole profile.

Although the use of the Sérsic profile may seem essential to our calculations, it is nothing but an analytical expression which works well from the theoretical point of view (essentially because it provides a shape factor) and also observationally (it gives good fits). In fact any other 
function would be equally appropriate - provided it allows to calculate the entropy, energy, mass, etc. This is not the case for the de Vaucouleurs law which is characterized by two parameters only, and for this reason does not allow to define the specific entropy or energy-mass surfaces. Consequently, it is not possible to define the EnergyEntropy line using the de Vaucouleurs law.

Notice that the de Vaucouleurs law is a homologous law, i.e., all models are deduced from a unique template by two scaling transformations. The introduction of a shape parameter in the Sérsic law destroys - mathematically speaking - this homology. However, since all galaxies fitted by a Sérsic law roughly have the same specific entropy, we can eliminate one parameter $\left(\Sigma_{0}\right.$, say) by replacing it with by its expression as function of the two remaining parameters, $\Sigma_{0}=\Sigma_{0}(a, \nu)$. Therefore we have - mathematically speaking - only two independent parameters, like the de Vaucouleurs law. This procedure, however, does not lead to any kind of homology, for it is not possible to define a "template" from which any Sérsic distribution could be derived only by scaling transformations.

Since all elliptical galaxies also obey the energy-mass relation, Eq. (10), we can further eliminate another parameter $(a$, say) with the result that a single parameter is sufficient to characterize a galaxy, for instance $\nu$. In this case every galaxy is defined by its shape parameter $\nu$ : generalizing the de Vaucouleurs law with the introduction of $\nu$ allows to gather all ellipticals into a single large family, at least from the photometric point of view.

The mass can then be rewritten formally as a function of $\nu$ only as:

$M(\nu)=\frac{2 \pi a^{2}(\nu)}{\nu} \Sigma_{0}(\nu) \mathcal{F} \Gamma\left(\frac{2}{\nu}\right)$,

where the equations for $a(\nu)$ and $\Sigma_{0}(\nu)$ are given in Eqs. (15) and (16). Moreover, very large galaxies are endowed with small values of $\nu$ while dwarfs have large values of $\nu$ (e.g., Caon et al. 1993, see also Paper I; the equation for $a(\nu)$ together with the definition of $R_{\text {eff }}$ also implies this result). Thus, the parameter $\nu$ plays the role of a concentration factor. This allows to understand why the de Vaucouleurs law cannot fit with good confidence these two extreme cases.

Equation (25) above is in fact a bijection $\nu \Longleftrightarrow M$. During the processes of formation and relaxation, physics (described by the two laws discussed above) are necessarily acting so that at the end the above bijection is actually verified.

\subsection{Distance indicators}

The interesting possibility of using the correlation between $[a, \nu]$ as a distance indicator has been proposed by Young \& Currie (1995). This paper has been followed by controversies (Binggeli \& Jerjen 1998; Young \& Currie 1998), essentially due, as we understand it, to the following question: are distance indicators sufficiently reliable to be usable for single galaxies, or can they only be applied to clusters globally?

Since the correlations on which are based the indicators proposed by Young \& Currie (1995) are consequences of the two laws discussed in this paper, it appears possible to improve their quality. This is because the predicted relations are essentially non-linear, while the observed correlations have been tentatively fitted by phenomenological linear laws, as we already pointed out.

This question has already been mentioned in Paper I and, in view of the predicted theoretical relations presented here, we therefore hope to improve the distance indicator based on the shape profile of Es (to be analysed in depth in a forthcoming paper for a dozen clusters with redshifts between 0.06 and 0.37 ).

\subsection{Conclusions}

We have shown both from theoretical reasons and from observations, that elliptical galaxies obey a scaling relation between the potential energy and mass (luminosity). In previous papers, we had already shown that Es share the same specific entropy (Papers I) with a logarithm dependence on the galactic mass (that may be due to an evolutionary process, e.g., hierarchical mergers Paper II).

These two relations, $U_{\mathrm{p}}-M$ and $s-\ln M$, give an explanation to several observed correlations that have been proposed in the past by various authors, such as the correlations between the shape factor and a length scale, the correlation between the absolute magnitude and the central brightness, and the photometric plane. Therefore they constitute a theoretical background for a number of physical properties of elliptical galaxies.

The fact that elliptical galaxies lie on a line in the three dimensional space of the Sérsic parameters implies that Es are indeed a one-parameter family. This has important implications for cosmology and galaxy formation and evolution models. Furthermore, the Energy-Entropy line could be used as a distance indicator.

Acknowledgements. We acknowledge interesting discussions with Gary Mamon. I.M. acknowledges financial support from the Spanish Ministerio de Educación y Cultura and the Instituto de Astrofísica de Andalucía (C.S.I.C.). This work is financed by DGICyT grants PB93-0139, PB96-0921, the USP/COFECUB and the CNPq/CNRS and C.S.I.C./CNRS bilateral cooperation agreements. G.B.L.N. acknowledges financial support from the FAPESP. B.L. is supported by a Marie Curie Training Grant (category 20), under the TMR Activity 3 of the European Community Program.

\section{Appendix A: Formulae}

General formulae useful for definitions or calculations of various quantities linked with our definition of the Sérsic profile are given in Tables A.1 and A.2. 
Table A.1. Various formulae related to the specific entropy and the energy-mass relation obtained for gravitational systems described by the Sérsic law. The 2-D and 3-D refer to the dependence on $\Sigma_{0}$ and $\rho_{0}$, respectively. The quantities $M^{*}, M_{3}^{*}$ and $r_{\mathrm{g}}^{*}$ are defined in Table A.2. Note the different normalization used for defining the specific entropy from Papers I and II.

\begin{tabular}{|l|l|}
\hline Quantity & Sérsic expression \\
\hline Specific entropy & $s=-\int \mathrm{d}^{3} r \mathrm{~d}^{3} v f^{*} \ln f^{*} ; \int \mathrm{d}^{3} r \mathrm{~d}^{3} v f^{*}=1$ \\
2-D expression & $s=\frac{3}{2} \ln \left(\mathcal{F}(L) \Sigma_{0}\right)+\frac{9}{2} \ln a+F_{2}(\nu) ;$ \\
& $F_{2}(\nu) \simeq-0.795 \ln (\nu)-\frac{1.34}{\nu}+3.85 \nu^{-1.29}+\ln \Gamma\left[\frac{2}{\nu}\right]-0.822$ \\
3-D expression & $s=\frac{3}{2} \ln \left(\mathcal{F}(L) j_{0}\right)+6 \ln a+F_{3} ;$ \\
& $F_{3}(\nu)=F_{2}(\nu)+\frac{3}{2} \ln \left[2 \frac{\Gamma([3-p] / \nu)}{\Gamma(2 / \nu)}\right]$ \\
Potential energy & $|U|=G a^{3}\left(2 \pi \mathcal{F} \Sigma_{0}\right)^{2} \times G_{2}^{*}(\nu) ;$ \\
2-D expression & $G_{2}^{*}(\nu) \simeq\left(\frac{\Gamma[2 / \nu]}{\nu \Gamma[(3-p) / \nu]}\right)^{2} \exp \left(1.7958 \nu^{-3 / 2}+2.6374 \sqrt{\nu}-5.4843\right)$ \\
Specific entropy-mass & $s=s_{0}+\delta_{s} \ln M$ \\
relation & $3 e_{0}=\ln \rho_{0}+\ln M_{3}^{*}(\nu)-3 \ln r_{\mathrm{g}}^{*}(\nu)+3 \ln G$ \\
2-D expression & \\
Energy-mass & \\
relation & $e_{0}=\ln U-\beta \ln M$ \\
& $3 e_{0}=\ln \Sigma_{0}-\ln a+\ln M_{2}^{*}(\nu)-3 \ln r_{\mathrm{g}}^{*}(\nu)+3 \ln G$ \\
\hline
\end{tabular}

The relation between the specific entropy and the mass, Eq. (2) can be expressed as a surface in the Sérsic parameters space with the help of the mass to light ratio, Eq. (11):

$$
\begin{aligned}
-\alpha^{\prime} \delta_{s}^{\prime} \ln \Sigma_{0}= & \delta_{s}^{\prime} \ln k_{F}+s_{0} \\
& +\left(\alpha^{\prime} \delta_{s}^{\prime}+\frac{3}{2}\right) \ln \left[\frac{2 \pi \Gamma(2 / \nu)}{\nu}\right]-F_{2}^{*}(\nu) \\
& +\left(2 \alpha^{\prime} \delta_{s}^{\prime}-\frac{3}{2}\right) \ln a
\end{aligned}
$$

where $\alpha^{\prime} \equiv \alpha+1, \delta_{s}^{\prime} \equiv \delta_{s}-3 / 2$ and the function $F_{2}^{*}(\nu)$ is defined in Table A.2.

The scaling relation between the potential energy and the mass gives another surface in the Sérsic parameters space:

$$
\begin{aligned}
-\alpha^{\prime} \beta^{\prime} \ln \Sigma_{0}= & \beta^{\prime} \ln k_{F}+\ln k_{U}-\ln \left(4 \pi^{2} G\right) \\
& +\left(\alpha^{\prime} \beta^{\prime}+2\right) \ln \left[\frac{2 \pi \Gamma(2 / \nu)}{\nu}\right]-\ln \left[G_{2}^{*}(\nu)\right] \\
& +\left[2\left(\alpha^{\prime} \beta^{\prime}+2\right)-3\right] \ln a,
\end{aligned}
$$

where $\alpha^{\prime} \equiv \alpha+1, \beta^{\prime} \equiv \beta-2$ and the function $G_{2}^{*}(\nu)$ is defined in Table A.2.

The intersection of the above two surfaces yields the locus where elliptical galaxies are found in the Sérsic parameter space. Eliminating $\Sigma_{0}$ from Eq. (A.1) or Eq. (A.2) we get:

$$
\begin{aligned}
\left(\frac{3}{2} \beta^{\prime}+\delta_{s}^{\prime}\right) \ln a= & \beta^{\prime}\left[s_{0}-F_{2}^{*}(\nu)\right] \\
& +\left(\frac{3}{2} \beta^{\prime}-2 \delta_{s}^{\prime}\right) \ln \left[\frac{2 \pi \Gamma(2 / \nu)}{\nu}\right] \\
& +\delta_{s}^{\prime} \ln \left[G 4 \pi^{2} G_{2}^{*}(\nu) / k_{U}\right]
\end{aligned}
$$

Note that $\ln a$ versus $\nu$ depends on the mass to light ratio only indirectly via $\delta_{s}, s_{0}, \beta$ and $e_{0}$. Likewise, eliminating $\ln a$ from the surface equations we obtain:

$$
\begin{aligned}
\left(\frac{3}{2} \beta^{\prime}+\delta_{s}^{\prime}\right) \alpha^{\prime} & \ln \Sigma_{0}=\left(2 \alpha^{\prime} \beta^{\prime}+1\right)\left(F_{2}^{*}[\nu]-s_{0}\right) \\
& -3\left(\left[\frac{3}{2} \beta^{\prime}-\delta_{s}^{\prime}\right] \alpha^{\prime}+\frac{3}{2}\right) \ln \left[\frac{2 \pi \Gamma(2 / \nu)}{\nu}\right] \\
& +\left(\frac{3}{2}-2 \alpha^{\prime} \delta_{s}^{\prime}\right) \ln \left[G 4 \pi^{2} G_{2}^{*}(\nu) / k_{U}\right] \\
& -\left(\frac{3}{2} \beta^{\prime}+\delta_{s}^{\prime}\right) \ln k_{F}
\end{aligned}
$$

The relation between $a$ and $\Sigma_{0}$ is obtained in parametric form combining the above two equations and using $\nu$ as a parameter. 
Table A.2. General formulae used in this paper. 2-D and 3-D quantities are marked with subscript "2" and " 3 ", respectively. Quantities marked with a "*” are adimensional. Some of these expressions were first obtained in Paper I and are presented here for completeness.

\begin{tabular}{|c|c|c|}
\hline Quantity & Theoretical Expression & Analytical Approximation \\
\hline \multicolumn{3}{|l|}{ Sérsic law } \\
\hline $2-\mathrm{D}$ & $\begin{array}{l}\Sigma(R)=\Sigma_{0} \exp \left(-(R / a)^{\nu}\right) \\
L_{2}(R)=a^{2} \Sigma_{0} \frac{2 \pi}{\nu} \gamma\left[\frac{2}{\nu},\left(\frac{R}{a}\right)^{\nu}\right]\end{array}$ & \\
\hline $3-\mathrm{D}$ & & $\begin{array}{l}j(r)=j_{0}\left(\frac{r}{a}\right)^{-p} \exp \left[-(r / a)^{\nu}\right] \\
p \simeq 1.0-0.6097 \nu+0.054635 \nu^{2} \\
L_{3}(r)=a^{3} j_{0} \frac{4 \pi}{\nu} \gamma\left[\frac{3-p}{\nu},\left(\frac{R}{a}\right)^{\nu}\right]\end{array}$ \\
\hline Mass profile & $M / L \equiv \mathcal{F}(L)=k_{F} L^{\alpha} ; \mathcal{F}(R)=$ constant & \\
\hline $2-\mathrm{D}$ & $M(R)=\mathcal{F}(L) L(R)$ & \\
\hline $3-\mathrm{D}$ & & $M(r)=\mathcal{F}(L) L_{3}(r)$ \\
\hline Total mass & $M=\mathcal{F}(L) L=k_{F} L^{\alpha+1}$ & \\
\hline $2-\mathrm{D}$ & $M=k_{F}\left(a^{2} \Sigma_{0} L_{2}^{*}\right)^{\alpha+1} ; L_{2}^{*}=\frac{2 \pi}{\nu} \Gamma\left[\frac{2}{\nu}\right]$ & \\
\hline $3-\mathrm{D}$ & & $M=k_{F}\left(a^{3} j_{0} L_{3}^{*}\right)^{\alpha+1} ; L_{3}^{*}=\frac{4 \pi}{\nu} \Gamma\left[\frac{3-p}{\nu}\right]$ \\
\hline Total "magnitude" & $\begin{aligned} \mathcal{M} & =-2.5 \log L \\
& =-2.5 \log \Sigma_{0}-5 \log a+m^{*}\end{aligned}$ & $m^{*} \simeq-0.30413 \nu-1.70786 \nu^{-1.44265}$ \\
\hline Mean $\mu$ inside $R_{\text {eff }}$ & $\begin{aligned}\langle\mu\rangle_{\mathrm{eff}} & =-2.5 \log \Sigma_{0}+m^{*}+ \\
& +5 \log \left(R_{\mathrm{eff}}^{*}\right)+2.5 \log 2 \pi\end{aligned}$ & \\
\hline Radii & $R_{i}=a \times R_{i}^{*}$ & \\
\hline Effec. Radius 3-D & $\gamma\left[(3-p) / \nu, R_{3, \mathrm{eff}}^{*}\right]=\frac{1}{2} \Gamma[(3-p) / \nu]$ & $\ln R_{3, \mathrm{eff}}^{*} \simeq \frac{0.72701-0.9877 \ln \nu}{\nu}+0.07021$ \\
\hline Effec. Radius 2-D & $\gamma\left(2 / \nu, R_{\mathrm{eff}}^{*}\right)=\frac{1}{2} \Gamma(2 / \nu)$ & $\ln R_{\mathrm{eff}}^{*} \simeq \frac{0.70348-0.99625 \ln \nu}{\nu}-0.18722$ \\
\hline Gravit. Radius & $\begin{array}{l}|U| \equiv G M^{2} / r_{g} \\
r_{g}^{*}=[\Gamma(2 / \nu) / \nu]^{2} / G_{2}^{*}(\nu)\end{array}$ & $\ln r_{\mathrm{g}}^{*} \simeq \frac{0.82032-0.92446 \ln \nu}{\nu}+0.84543$ \\
\hline$r_{\mathrm{g}}$ versus $R_{\mathrm{eff}}$ & $a \times j_{0}=\Sigma_{0} \frac{L_{2}^{*}}{I^{*}}$ & $\ln r_{\mathrm{g}}^{*} \simeq 1.16+0.98 \ln R_{\mathrm{eff}}^{*}$ \\
\hline$\Sigma_{0} \Leftrightarrow j_{0}$ & $a \times j_{0}=\Sigma_{0} \times \frac{\Gamma(2 / \nu)}{2 \Gamma([3-p] / \nu)}$ & $\begin{array}{l}a j_{0} \simeq \Sigma_{0} \times \\
\times\left(0.076685+0.3253 \nu-0.041245 \nu^{2}\right)\end{array}$ \\
\hline
\end{tabular}

\section{References}

Bertin, G., Saglia, R. P., \& Stiavelli, M. 1992, ApJ, 384, 423 Binggeli, B., Sandage, A., \& Tarenghi, M. 1984, AJ, 89, 64 Binggeli, B., \& Jerjen, H. 1998, A\&A, 333, 17

Burstein, D., Bender, R., Faber, S. M., \& Nolthenius, R. 1997, AJ, 114, 1365

Caon, N., Capaccioli, M., \& D'Onofrio, M. 1993, MNRAS, 265, 1013

Capelato, H. V., de Carvalho, R. R., \& Carlberg, R. G. 1995, ApJ, 451, 525

Capelato, H. V., de Carvalho, R. R., \& Carlberg, R. G. 1997, in Galaxy Scaling Relations: Origins, Evolution and Applications, ed. L. N. da Costa, \& A. Renzini (SpringerVerlag), 331

Djorgovski, S., \& Davis, M. 1987, ApJ, 313, 59
Dressler, A., Lynden-Bell, D., Burstein, D., et al. 1987, ApJ, 313,42

Durret, F., Demarco, R., Magnard, F., \& Gerbal, D. 2001, Rencontres de Moriond, March 10-17, 2001, Les Arcs (Savoie), ed. D. Neumann et al. [astro-ph/0105496]

Faber, S. M., \& Jackson, R. E. 1976, ApJ, 204, 668

Fadda, D., Slezak, E., \& Bijaoui, A. 1998, A\&AS, 127, 335

Feigelson, E. D., \& Babu, G. J. 1992, ApJ, 397, 55

Fish, R. A. 1964, ApJ, 139, 284

Gerbal, D., Lima Neto, G. B., Márquez, I., \& Verhagen, H. 1997, MNRAS, 285, L41

Gerhard, O., Kronawitter, A., Saglia, R. P., \& Bender, R. 2001, AJ, 121, 1936

Graham, A. 1998, MNRAS, 295, 933

Graham, A., \& Colless, M. 1997, MNRAS, 287, 221

Griffiths, R. E., Casertano, S., Im M., \& Ratnatunga, K. U. 1998, MNRAS, 282, 1159 
Jang-Condell, H., \& Hernquist, L. 2001, ApJ, 548, 68

Jerjen, H., \& Binggeli, B. 1997, in The Nature of Elliptical Galaxies, proceedings of the Second Stromlo Symposium, ed. M. Arnaboldi et al., ASP Conf. Ser., 116, 239

Jorgensen, I., Franx, M., Hjorth, J., \& van Dokkum, P. G. 1999, MNRAS, 308, 833

Kauffmann, G., Colberg, J. M., Diaferio, A., \& White, S. D. M. 1999, MNRAS, 303, 188

Khosroshahi, H. G., Wadadekar, Y., Kembhavi, A., \& Mobasher, B. 2000, ApJ, 531, L103

Kormendy, J. 1977, ApJ, 218, 333

Kormendy, J., \& Richstone, D. 1995, ARAA, 33, 581

Kronawitter, A., Saglia, R. P., Gerhard, O., \& Bender, R. 2000, A\&AS, 144, 53

Lima Neto, G. B., Gerbal, D., \& Márquez, I. 1999, MNRAS, 309, 481 (Paper I)
Loewenstein, M., \& White, R. E. 1999, ApJ, 518, 50

Márquez, I., Lima Neto, G. B., Capelato, H., Durret, F., \& Gerbal, D. 2000, A\&A, 353, 873 (Paper II)

Merritt, D. 1999, PASP, 111, 129

Prugniel, Ph., \& Simien, F. 1997, A\&A, 321, 111

Saglia, R. P., Bertschinger, E., Baggley, G., et al. 1997, ApJS, 109, 79

Schombert, J. M. 1987, ApJS, 64, 643

Sérsic, J. L. 1968, Atlas de galaxias australes, Observatorio Astronómico de Córdoba, Argentina

Yoshida, N., \& the Virgo Consortium 2001, in preparation

Young, C. K., \& Currie, M. J. 1994, MNRAS, 268, L11

Young, C. K., \& Currie, M. J. 1995, MNRAS, 273, 1141

Young, C. K., \& Currie, M. J. 1998, A\&A, 333, 795

de Zeeuw, T., \& Franx, M. 1991, ARAA, 29, 275 